\title{
Association of attention-deficit/hyperactivity disorder with diabetes: a population-based study
}

\author{
Hui-Ju Chen ${ }^{1,2}$, Yann-Jinn Lee ${ }^{1,3,4}$, Geng Chang Yeh ${ }^{1,5}$ and Herng-Ching Lin ${ }^{6}$
}

BACKGROUND: Cognitive impairment has been documented in adult diabetes but is unclear in pediatric diabetes. No study had been conducted to explore the relationship between attention-deficit/hyperactivity disorder (ADHD) and diabetes. Using a population-based data set, we aimed to examine the association between ADHD and a prior diagnosis of diabetes mellitus (DM) in Taiwan.

METHODS: A total of 4,302 patients with ADHD were selected as cases and 21,510 randomly selected subjects as controls. We used conditional logistic regression to calculate the odds ratio (OR) for having previously received a diagnosis of DM between subjects with and without ADHD.

RESULTS: In this study, 116 of the 25,812 sampled subjects (0.5\%) had received a diagnosis of DM prior to their index date. Subjects with ADHD had a higher proportion of prior DM diagnoses than controls ( $0.9 \%$ vs. $0.4 \%, P<0.001)$. After adjusting for age, sex, index year, geographic location, and obesity, ADHD was significantly associated with a prior diagnosis of type $2 \mathrm{DM}(\mathrm{OR}=2.75$, 95\% confidence interval $(\mathrm{Cl})=1.82-4.16)$. However, no significant association was observed between ADHD and type $1 \mathrm{DM}$.

CONCLUSION: The findings suggest that ADHD was associated with a previous diagnosis of type $2 \mathrm{DM}$.

A ttention-deficit/hyperactivity disorder (ADHD) is the most common cognitive and behavioral disorder diagnosed among schoolchildren and adolescents, with an estimated prevalence of $5.29 \%$ (1). The pathophysiology of ADHD is marked by dopaminergic and nonadrenergic dysregulation, as well as by structural and functional abnormalities of the cortico-cortical and fronto-subcortical pathways, including those of the striatum and cerebellum (2). The etiology of ADHD is multifactorial and generally stems from a combination of genetic and acquired factors. Various biological and environmental factors, including viral infection (3), lead contamination (4), maternal smoking during pregnancy (5), alcohol exposure (6), prematurity (7), low birth weight (8), and nutritional and thyroid disorders (9), may contribute to its development. However, it is still not clear whether diabetes mellitus (DM) is a risk factor for the development of ADHD among children.
The effects of DM on the central nervous system have been emphasized recently. In studies conducted among school-aged children, those with early-onset DM have been shown to demonstrate poorer academic achievement, visual spatial ability, memory, motor speed, and eye-hand coordination $(10,11)$. Type 2 DM, known as a common disorder in adulthood, is dramatically increasing in both children and adolescents, in relation to increased incidence of obesity (12). Some psychiatric disorders, such as depression and behavior disorders, are reported to be increased in patients with type $2 \mathrm{DM}(13,14)$. It has been suggested that chronic hyperglycemia at an early age increases neuronal vulnerability $(15,16)$ and may impede myelination of the developing brain (17). The results of animal and human case studies suggest that hypoglycemia may induce cell death in the brain, possibly through excitotoxic or apoptotic processes $(18,19)$. However, although both chronic hyperglycemia and recurrent episodes of hypoglycemia may lead to central nervous system damage, the long-term effects of DM on the central nervous system are still controversial $(20,21)$. To the best of our knowledge, no study has attempted to explore the association between ADHD and DM. Therefore, using a population-based data set, we aimed to examine the association between ADHD and prior DM diagnosis in Taiwan.

\section{RESULTS}

This study included 4,302 newly diagnosed subjects with ADHD and 21,510 matched controls. Of the 25,812 sampled subjects, the mean age was $8.6 \mathrm{y}(\mathrm{SD}=2.7 \mathrm{y})$. Table 1 shows the distribution of demographic characteristics between subjects with and without ADHD. After matching for age and sex, subjects with ADHD were found to more likely be residents of the northern part of Taiwan $(P<0.001)$ than subjects without ADHD.

Table 2 presents the prevalence of prior diabetes between subjects with and without ADHD. It shows that 116 of the $25,812$ sampled subjects ( $0.5 \%)$ were diagnosed with diabetes prior to the index date. Subjects with ADHD had a higher proportion of prior diabetes as compared with subjects without ADHD ( 0.9 vs. $0.4 \%, P<0.001)$. We further analyzed the prevalence of type $1 \mathrm{DM}$ and type $2 \mathrm{DM}$ between subjects with and without ADHD. We found that subjects with ADHD had a 
Table 1. Demographic characteristics of patients with attentiondeficit/hyperactivity disorder and controls in Taiwan in the years 2002-2008 $(N=25,812)$

\begin{tabular}{|c|c|c|c|c|c|}
\hline \multirow[b]{2}{*}{ Variable } & \multicolumn{2}{|c|}{$\begin{array}{l}\text { Patients with } \\
\text { attention-deficit/ } \\
\text { hyperactivity } \\
\text { disorder }(n=4,302)\end{array}$} & \multicolumn{2}{|c|}{$\begin{array}{c}\text { Controls } \\
(n=21,510)\end{array}$} & \multirow[b]{2}{*}{$P$ value } \\
\hline & Total no. & $\%$ & Total no. & $\%$ & \\
\hline Age (years) & & & & & 1.000 \\
\hline 5 & 474 & 11.0 & 2,370 & 11.0 & \\
\hline 6 & 627 & 14.6 & 3,135 & 14.6 & \\
\hline 7 & 755 & 17.6 & 3,775 & 17.6 & \\
\hline 8 & 578 & 13.4 & 2,890 & 13.4 & \\
\hline 9 & 469 & 10.9 & 2,345 & 10.9 & \\
\hline 10 & 391 & 9.1 & 1,955 & 9.1 & \\
\hline 11 & 278 & 6.5 & 1,390 & 6.5 & \\
\hline 12 & 247 & 5.7 & 1,235 & 5.7 & \\
\hline 13 & 232 & 5.4 & 1,160 & 5.4 & \\
\hline 14 & 168 & 3.9 & 840 & 3.9 & \\
\hline 15 & 83 & 1.9 & 415 & 1.9 & \\
\hline Sex & & & & & 1.000 \\
\hline Male & 3,442 & 80.0 & 17,210 & 80.0 & \\
\hline Female & 860 & 20.0 & 4,300 & 20.0 & \\
\hline Geographic region & & & & & $<0.001$ \\
\hline Northern Taiwan & 2,473 & 57.5 & 10,040 & 46.7 & \\
\hline Central Taiwan & 737 & 17.1 & 5,446 & 25.3 & \\
\hline Southern Taiwan & 997 & 23.2 & 5,514 & 25.6 & \\
\hline Eastern Taiwan & 95 & 2.2 & 510 & 2.4 & \\
\hline Obesity & 23 & 0.5 & 56 & 0.3 & 0.003 \\
\hline
\end{tabular}

Table 2. Prevalence for prior diabetes among the sampled patients

\begin{tabular}{|c|c|c|c|c|c|c|}
\hline \multirow[b]{2}{*}{ Variable } & \multicolumn{2}{|c|}{$\begin{array}{c}\text { Total } \\
(N=25,812)\end{array}$} & \multicolumn{2}{|c|}{$\begin{array}{l}\text { Patients with attention- } \\
\text { deficit/hyperactivity } \\
\text { disorder }(n=4,302)\end{array}$} & \multicolumn{2}{|c|}{$\begin{array}{c}\text { Controls } \\
(n=21,510)\end{array}$} \\
\hline & $n$ & $\%$ & $n$ & $\%$ & $n$ & $\%$ \\
\hline \multicolumn{7}{|c|}{ Presence of diabetes } \\
\hline Yes & 116 & 0.5 & 40 & 0.9 & 76 & 0.4 \\
\hline \multicolumn{7}{|c|}{ Presence of type 1 diabetes } \\
\hline Yes & 16 & 0.1 & 4 & 0.1 & 12 & 0.1 \\
\hline \multicolumn{7}{|c|}{ Presence of type 2 diabetes } \\
\hline Yes & 100 & 0.4 & 36 & 0.8 & 64 & 0.3 \\
\hline
\end{tabular}

higher prevalence of type $2 \mathrm{DM}$ than subjects without ADHD $(0.8$ vs. $0.3 \%, P<0.001)$. However, there was no significant difference in the prevalence of type $1 \mathrm{DM}$ between subjects with and without $\mathrm{ADHD}(0.1$ vs. $0.1 \%, P=0.371)$.

Table 3 shows the crude and covariate-adjusted odds ratios (ORs) and 95\% confidence intervals (CIs) for ADHD. Conditional logistic regression suggested that $\mathrm{ADHD}$ was significantly related to type $2 \mathrm{DM}(\mathrm{OR}=2.83,95 \% \mathrm{CI}=1.88-4.26)$ but not type $1 \mathrm{DM}(\mathrm{OR}=1.68,95 \% \mathrm{CI}=0.54-5.20)$. Furthermore,
Table 3. Crude and covariate-adjusted ORs for attention-deficit/ hyperactivity disorder among the sampled patients $(N=25,812)$

\begin{tabular}{|c|c|c|}
\hline \multirow[b]{2}{*}{ Variable } & \multicolumn{2}{|c|}{ Attention-deficit/hyperactivity disorder } \\
\hline & Crude OR $(95 \% \mathrm{Cl})$ & Adjusted OR (95\% Cl) \\
\hline \multicolumn{3}{|l|}{ DM } \\
\hline Type 1 & $1.68(0.54-5.20)$ & $1.62(0.51-5.14)$ \\
\hline Type 2 & $2.83^{+}(1.88-4.26)$ & $2.75^{\dagger}(1.82-4.16)$ \\
\hline No (reference group) & 1.00 & 1.00 \\
\hline \multicolumn{3}{|l|}{ Age (years) } \\
\hline 5 (reference group) & 1.00 & 1.00 \\
\hline 6 & $1.00(0.88-1.14)$ & $1.00(0.88-1.14)$ \\
\hline 7 & $1.00(0.88-1.13)$ & $1.00(0.88-1.14)$ \\
\hline 8 & $1.00(0.88-1.14)$ & $0.99(0.87-1.14)$ \\
\hline 9 & $1.00(0.87-1.15)$ & $1.01(0.87-1.15)$ \\
\hline 10 & $1.00(0.86-1.16)$ & $1.00(0.86-1.16)$ \\
\hline 11 & $1.00(0.85-1.18)$ & $1.01(0.86-1.18)$ \\
\hline 12 & $1.00(0.85-1.18)$ & $1.01(0.86-1.18)$ \\
\hline 13 & $1.00(0.85-1.19)$ & $1.00(0.85-1.19)$ \\
\hline 14 & $1.00(0.83-1.21)$ & $1.00(0.84-1.23)$ \\
\hline 15 & $1.00(0.78-1.29)$ & $1.01(0.79-1.32)$ \\
\hline \multicolumn{3}{|l|}{ Index year } \\
\hline 2002 (reference group) & 1.00 & 1.00 \\
\hline 2003 & $1.00(0.87-1.13)$ & $1.01(0.88-1.14)$ \\
\hline 2004 & $1.00(0.86-1.16)$ & $1.01(0.88-1.16)$ \\
\hline 2005 & $1.00(0.87-1.14)$ & $1.00(0.87-1.13)$ \\
\hline 2006 & $1.00(0.89-1.10)$ & $1.01(0.87-1.16)$ \\
\hline 2007 & $1.00(0.86-1.16)$ & $0.99(0.86-1.16)$ \\
\hline 2008 & $1.00(0.87-1.17)$ & $1.00(0.87-1.18)$ \\
\hline \multicolumn{3}{|l|}{ Sex } \\
\hline Male & $1.00(0.92-1.09)$ & $0.99(0.92-1.08)$ \\
\hline Female (reference group) & 1.00 & 1.00 \\
\hline Obesity & $2.16^{* *}(1.26-3.71)$ & $1.98^{*}(1.15-3.40)$ \\
\hline
\end{tabular}

$\mathrm{Cl}$, confidence interval; $\mathrm{DM}$, diabetes mellitus; $\mathrm{OR}$, odds ratio.

${ }^{*} P<0.05 ;{ }^{*} P<0.01 ;{ }^{\dagger} P<0.001$.

after adjusting for age, sex, index year, geographic location, and obesity, ADHD was significantly associated with prior type 2 $\mathrm{DM}(\mathrm{OR}=2.75,95 \% \mathrm{CI}=1.82-4.16)$. However, no significant association was detected between ADHD and type $1 \mathrm{DM}$.

\section{DISCUSSION}

In this investigation, we found type $2 \mathrm{DM}$ to be associated with ADHD after adjusting for potential confounding factors. To the best of our knowledge, this is the first study to demonstrate this association. The pathogenesis of type $2 \mathrm{DM}$ is quite different from that of type $1 \mathrm{DM}$. Type $2 \mathrm{DM}$ is primarily caused by insulin resistance on target tissues and relative insulin deficiency, whereas type $1 \mathrm{DM}$ is mainly caused by insulin deficiency due to the autoimmune-mediated destruction of pancreatic $\beta$ cells (22). The incidence of type $2 \mathrm{DM}$ due to obesity and associated insulin resistance in childhood and 
adolescence is dramatically increasing. It is estimated that up to $45 \%$ of all DM reported in childhood and adolescence is type 2 DM $(12,23)$. Although there is significant emerging literature regarding the adverse effects of type $2 \mathrm{DM}$ on the central nervous system in middle-aged and elderly individuals (24-26), there is little information available regarding this effect among the pediatric group. Adults with type $2 \mathrm{DM}$ have been found to have decreasing white matter volumes, especially in the frontal area, and cortical/subcortical atrophy $(27,28)$. The impairment of cognitive function in patients with type $2 \mathrm{DM}$ has also been demonstrated to be correlated to the reduced circulation of the frontal and parietal lobes (29). The hippocampus and frontal area are regions responsible for attention, cognition, and motor planning. Damage to these regions may lead to inattention, loss of emotion, behavioral inhibition, and poor memory.

$\mathrm{ADHD}$ is characterized by impairments in attention, motor, and emotion regulation. According to various neuroimaging and functional studies, the core of the attention network includes the lateral and medial prefrontal cortices, the lateral-inferior parietal and temporo-occipito-parietal cortices in the surface of the right hemisphere $(30,31)$, and the circuits of the cortico-striatal and cortico-cerebellar networks. These subserve motor, cognitive, and emotional behaviors (32). Type 2 DM most commonly affects the frontal and hippocampus regions, which may explain the risk for developing ADHD symptoms later.

Of interest, type $1 \mathrm{DM}$ was not shown to be significantly associated with ADHD in our study. Rovet and Alvarez (33) have reported that attentional cognitive processes are disrupted in children and adolescents with type 1 DM, but no other study has been conducted investigating the correlation of executive symptoms, the core symptom of ADHD, with DM. One possible reason is that type $1 \mathrm{DM}$ and type $2 \mathrm{DM}$ demonstrate different pathophysiologies of insulin regulation. Type 2 DM is characterized by insulin resistance on target tissues such as the brain. Altfas (34) reported that ADHD was highly prevalent among obese patients and highest in those with extreme obesity. Bruehl et al. (35) reported that adolescents with type 2 DM with insulin resistance have significantly reduced hippocampal and prefrontal volumes and higher rates of global cerebral atrophy as compared with obese controls without insulin resistance. Insulin and insulin receptor signaling play a modulatory role in learning and memory processing (36). The role of hyperinsulinemia and abnormalities in insulin signaling within the brain need to be studied because they might explain some of the apparent differential effects of type 1 diabetes and type 2 diabetes on the brain.

The primary strength of our study lies in its population-based database and large sample population, which mitigate the effect of the selection biases inherent in studies utilizing data taken from voluntary registries and hospital-referred study patients. However, this study suffered from several limitations that should be addressed. The first is the use of International Classification of Disease, 9th edition (ICD-9) coding in the administrative database to diagnose ADHD and DM. Because of this we were unable to include any measure of severity of ADHD and DM in this investigation. Second, the Longitudinal Health Insurance Database (LHID2005) lacks information on prenatal, perinatal social, parental psychopathological variables, and body weight information that have been reported to be associated with ADHD (37). Third, surveillance bias could have been a potential limitation if patients with DM were more likely to be screened for ADHD. However, this study found that $\mathrm{ADHD}$ was associated only with type $2 \mathrm{DM}$, not type 1 DM. This accordingly discounts the possibility that receiving medical attention for DM contributed to elevated odds of developing ADHD. Finally, although we have controlled for comorbid psychotic and mood disorders, sometimes ADHD behaviors are extreme enough that a physician may prescribe an antipsychotic medication. As these medications have been suggested to contribute to diabetes, it is possible that their use influenced the results of this study. This avenue of investigation should be further explored in future studies (38).

In conclusion, our report suggests that type $2 \mathrm{DM}$ in childhood is associated with ADHD. Type $2 \mathrm{DM}$ is a chronic medical condition with an increasing childhood and adolescent incidence, during crucial times for brain development. ADHD is a neurological disorder with high risk of adverse personal and social outcomes. Early identification of children at risk for ADHD among patients with DM is necessary to ensure that they receive prompt and appropriate treatment. Primary physicians should provide routine screening for ADHD in addition to routine primary diabetic management.

\section{METHODS \\ Database}

The data for this study were sourced from the LHID2005. The LHID2005 was created and released to the public by the National Health Research Institute and includes all the original claim data and registration files for $1,000,000$ individuals randomly sampled from the 2005 Registry for Beneficiaries ( $n=25.68$ million) of the National Health Insurance program. The Taiwan National Health Research Institute has validated the representativeness of the LHID2005 relative to the whole population of National Health Insurance enrollees according to gender distribution, age distribution, and average insured payroll-related amount. The LHID2005 provided an exclusive opportunity for researchers to trace all the medical utilizations of these $1,000,000$ enrollees since the inauguration of the National Health Insurance program.

Because the LHID2005 consists of de-identified secondary data released to the public for research purposes, this study was exempted from full review by the Taipei Medical University Institutional Review Board.

\section{Selection of Cases and Controls}

This study was designed as a case-control study. The case-control study is frequently contrasted with cohort studies, in which exposed and unexposed subjects are observed until they develop an outcome of interest. Therefore, this study did not study the incident cases of DM, and the precise causal relationship between ADHD and DM cannot be determined by the case-control study. We selected 5,554 patients aged between 5 and $15 \mathrm{y}$ who received a first-time diagnosis of ADHD (ICD-9-Clinical Modification (CM) (39) code 314.00 or 314.01) in their ambulatory care visits between January 2002 and December 2008). To increase the diagnostic validity of the subjects with ADHD in this study, we included only those cases who had received $\geq 3$ ADHD diagnoses. In Taiwan, the first diagnosis of a condition reflects the clinician's utilization of a diagnostic test to assess the condition in 
question, whereas the second diagnosis indicates the presence of the condition based on the outcome of both the diagnostic test and a clinical examination. Therefore, by limiting our cases to those who received $\geq 3$ ADHD diagnoses, we assured that all of our cases received a positive diagnosis based on their symptoms, medical history, and the results of at least one diagnostic test. We excluded patients who had been diagnosed with preterm births (ICD-9-CM codes 765.00-765.09, 765.10765.19) or low birth weight (ICD-9-CM codes V21.30-V21.35) $(n=$ $820)$, which have been reported to be risk factors for $\operatorname{ADHD}(7,8)$. In addition, we excluded patients who had been diagnosed with a congenital anomaly (ICD-9-CM codes 758.0-758.9, 759.9) $(n=41)$. We further excluded subjects who had been diagnosed with mental illness (ICD-9-CM codes 290-319, except 314.01) $(n=20)$. We also excluded patients who had received a diagnosis of cerebral palsy, intracranial hemorrhage, viral encephalitis, hypoxic-ischemic encephalitis, epilepsy/seizure, or thyroid disorder $(n=371)$. Ultimately, 4,302 patients with ADHD were selected as cases. We assigned the date of their first diagnosis of ADHD as the index date.

We likewise selected five controls for each ADHD case, based upon the principles provided by Hennessy et al. (40), from the remaining enrollees of the LHID2005. A total of 21,510 controls were selected. These controls were frequency-matched with cases by age, sex, and index year. We assured that none of the selected controls had ever been diagnosed with any type of mental illness, preterm birth, low birth weight, congenital anomaly, cerebral palsy, intracranial hemorrhage, viral encephalitis, hypoxic-ischemic encephalitis, epilepsy/seizure, or thyroid disorder. For controls, we assigned their first use of ambulatory care occurring in the index year as their index date.

\section{Exposure Assessment}

We identified diabetes cases based on ICD-9-CM code 250 (22). To increase the diagnostic validity of diabetes, we included only patients who had at least two consensus diabetes diagnoses. Furthermore, this study included diabetes cases only if the diabetes diagnosis was made before the index date.

\section{Statistical Analysis}

We used SAS system (SAS System for Windows, Version 8.2; SAS Institute, Cary, NC) for the statistical analyses. We first compared the distributions of the geographic location of the community in which the patient resided (northern, central, eastern, or southern Taiwan) between cases and controls using $\left({ }^{2}\right.$ tests. We used logistic regression (conditioned on age, sex, and index year) to explore the relationship between diabetes and ADHD. In the regression models, we also adjusted for age, sex, index year, geographic region, and obesity (ICD9-CM codes 278, 278.0, 278.00, and 278.01). In this study, although we identified obesity cases by ICD-9-CM codes, obesity for children (aged $2-19$ ) is defined as a body mass index at or above the 95th percentile for children of the same age and sex in Taiwan. A $P$ value of $\leq 0.05$ was used to assess statistical significance in this study.

\section{ACKNOWLEDGMENTS}

This study is based in part on data from the National Health Insurance Research Database provided by the Bureau of National Health Insurance, Department of Health, Taiwan and managed by the National Health Research Institutes. The interpretations and conclusions contained herein do not represent those of the Bureau of National Health Insurance, Department of Health, or the National Health Research Institutes.

Disclosure: The authors declared no conflict of interest.

\section{REFERENCES}

1. Polanczyk G, de Lima MS, Horta BL, Biederman J, Rohde LA. The worldwide prevalence of ADHD: a systematic review and metaregression analysis. Am J Psychiatry 2007;164:942-8.

2. Biederman J. Attention-deficit/hyperactivity disorder: a selective overview. Biol Psychiatry 2005;57:1215-20.

3. Millichap JG. Encephalitis virus and attention deficit hyperactivity disorder. J R Soc Med 1997;90:709-10.

4. Needleman HL. Lead and impaired abilities. Dev Med Child Neurol 1982;24:196-8.
5. Huizink AC, Mulder EJ. Maternal smoking, drinking or cannabis use during pregnancy and neurobehavioral and cognitive functioning in human offspring. Neurosci Biobehav Rev 2006;30:24-41.

6. Knopik VS, Sparrow EP, Madden PA, et al. Contributions of parental alcoholism, prenatal substance exposure, and genetic transmission to child ADHD risk: a female twin study. Psychol Med 2005;35:625-35.

7. Lindström K, Lindblad F, Hjern A. Preterm birth and attention-deficit/ hyperactivity disorder in schoolchildren. Pediatrics 2011;127:858-65.

8. Mick E, Biederman J, Prince J, Fischer MJ, Faraone SV. Impact of low birth weight on attention-deficit hyperactivity disorder. J Dev Behav Pediatr 2002;23:16-22.

9. Spencer T, Biederman J, Wilens T, Guite J, Harding M. ADHD and thyroid abnormalities: a research note. J Child Psychol Psychiatry 1995;36:879-85.

10. Desrocher M, Rovet J. Neurocognitive correlates of type 1 diabetes mellitus in childhood. Child Neuropsychol 2004;10:36-52.

11. Gaudieri PA, Chen R, Greer TF, Holmes CS. Cognitive function in children with type 1 diabetes: a meta-analysis. Diabetes Care 2008;31:1892-7.

12 Type 2 diabetes in children and adolescents. American Diabetes Association. Diabetes Care 2000;23:381-9.

13. Levitt Katz LE, Swami S, Abraham M, et al. Neuropsychiatric disorders at the presentation of type 2 diabetes mellitus in children. Pediatr Diabetes 2005;6:84-9.

14. Block WM, Putzer GJ, Jaramillo JR. Children with type 2 diabetes mellitus and the prevalence of psychiatric disorders. South Med J 2010;103:1214-8

15. Malone JI, Hanna S, Saporta S, et al. Hyperglycemia not hypoglycemia alters neuronal dendrites and impairs spatial memory. Pediatr Diabetes 2008;9:531-9.

16. Malone JI, Hanna SK, Saporta S. Hyperglycemic brain injury in the rat. Brain Res 2006;1076:9-15.

17. Vlassara H, Brownlee M, Cerami A. Excessive nonenzymatic glycosylation of peripheral and central nervous system myelin components in diabetic rats. Diabetes 1983;32:670-4.

18. Fujioka M, Okuchi K, Hiramatsu KI, Sakaki T, Sakaguchi S, Ishii Y. Specific changes in human brain after hypoglycemic injury. Stroke 1997;28:584-7.

19. Auer RN, Olsson Y, Siesjö BK. Hypoglycemic brain injury in the rat. Correlation of density of brain damage with the EEG isoelectric time: a quantitative study. Diabetes 1984;33:1090-8.

20. Ryan CM. Why is cognitive dysfunction associated with the development of diabetes early in life? The diathesis hypothesis. Pediatr Diabetes 2006; 7:289-97.

21. Sima AA, Kamiya H, Kamiya H, Li ZG. Insulin, C-peptide, hyperglycemia, and central nervous system complications in diabetes. Eur J Pharmacol 2004;490:187-97.

22. Stumvoll M, Goldstein BJ, van Haeften TW. Type 2 diabetes: principles of pathogenesis and therapy. Lancet 2005;365:1333-46.

23. Aye T, Levitsky LL. Type 2 diabetes: an epidemic disease in childhood. Curr Opin Pediatr 2003;15:411-5.

24. Bruehl H, Wolf OT, Sweat V, Tirsi A, Richardson S, Convit A. Modifiers of cognitive function and brain structure in middle-aged and elderly individuals with type 2 diabetes mellitus. Brain Res 2009;1280:186-94.

25. Ryan CM, Geckle M. Why is learning and memory dysfunction in Type 2 diabetes limited to older adults? Diabetes Metab Res Rev 2000;16:308-15.

26. Awad N, Gagnon M, Messier C. The relationship between impaired glucose tolerance, type 2 diabetes, and cognitive function. J Clin Exp Neuropsychol 2004;26:1044-80.

27. Hsu JL, Chen YL, Leu JG, et al. Microstructural white matter abnormalities in type 2 diabetes mellitus: a diffusion tensor imaging study. Neuroimage 2012;59:1098-105.

28. Novak V, Last D, Alsop DC, et al. Cerebral blood flow velocity and periventricular white matter hyperintensities in type 2 diabetes. Diabetes Care 2006;29:1529-34.

29. Niwa H, Koumoto C, Shiga T, et al. Clinical analysis of cognitive function in diabetic patients by MMSE and SPECT. Diabetes Res Clin Pract 2006;72:142-7.

30. Duncan J, Owen AM. Common regions of the human frontal lobe recruited by diverse cognitive demands. Trends Neurosci 2000;23:475-83. 


\section{Articles $\mid$ Chen et al.}

31. Goldman-Rakic PS. Topography of cognition: parallel distributed networks in primate association cortex. Annu Rev Neurosci 1988;11:137-56.

32. Makris N, Meyer JW, Bates JF, Yeterian EH, Kennedy DN, Caviness VS. MRI-Based topographic parcellation of human cerebral white matter and nuclei II. Rationale and applications with systematics of cerebral connectivity. Neuroimage 1999;9:18-45.

33. Rovet J, Alvarez M. Attentional functioning in children and adolescents with IDDM. Diabetes Care 1997;20:803-10.

34. Altfas JR. Prevalence of attention deficit/hyperactivity disorder among adults in obesity treatment. BMC Psychiatry 2002;2:9.

35. Bruehl H, Sweat V, Tirsi A, Shah B, Convit A. Obese adolescents with type 2 diabetes mellitus have hippocampal and frontal lobe volume reductions. Neurosci Med 2011;2:34-42.
36. Dou JT, Chen M, Dufour F, Alkon DL, Zhao WQ. Insulin receptor signaling in long-term memory consolidation following spatial learning. Learn Mem 2005;12:646-55.

37. Galéra C, Côté SM, Bouvard MP, et al. Early risk factors for hyperactivityimpulsivity and inattention trajectories from age 17 months to 8 years. Arch Gen Psychiatry 2011;68:1267-75.

38. Andrade SE, Lo JC, Roblin D, et al. Antipsychotic medication use among children and risk of diabetes mellitus. Pediatrics 2011;128:1135-41.

39. Carol J, Buck CJ. W.B. Saunders 2001 ICD-9-CM, volumes 1, 2, and 3. Singapore: Elsevier (Singapore), 2003:637, 139-40, 368-72.

40. Hennessy S, Bilker WB, Berlin JA, Strom BL. Factors influencing the optimal control-to-case ratio in matched case-control studies. Am J Epidemiol 1999;149:195-7. 